\title{
THE RELATIVE GROWTH OF INFORMATION IN TWO-DIMENSIONAL PARTITIONS
}

KARMA DAJANI, MARTIJN DE VRIES, AND AIMEE S. A. JOHNSON

\begin{abstract}
Let $\bar{x} \in[0,1)^{2}$. In this paper we find the rate at which knowledge about the partition elements $\bar{x}$ lies in for one sequence of partitions determines the partition elements it lies in for another sequence of partitions. This rate depends on the entropy of these partitions and the geometry of their shapes, and gives a two-dimensional version of Lochs' Theorem.
\end{abstract}

Date: November 15, 2004.

1991 Mathematics Subject Classification. Primary:28D15; Secondary:28D20.

Key words and phrases. Lochs' Theorem, Shannon-McMillan-Breiman Theorem.

The research of the third author was partially supported by the NSF-AWM Michler collaborative Reserach Grant, NWO bezoekersbeurs no B 61-547, and the Michner grant. 


\section{INTRODUCTION}

Let $x \in[0,1)$ and suppose we are interested in 2 different numbertheoretic expansions of $x$. Given $n$ digits in one of the expansions, how many digits are determined in the other expansion?

In 1964, G. Lochs explored this question when comparing the decimal and continued fraction expansions of $x$. Let $x \in[0,1)$ be irrational with decimal expansion

$$
x=. d_{1} d_{2} d_{3} \ldots
$$

and continued fraction expansion

$$
x=\frac{1}{a_{1}+\frac{1}{a_{2}+\frac{1}{a_{3}+\cdots}}}=\left[0 ; a_{1}, a_{2}, a_{3}, \cdots\right]
$$

If only the first $n$ decimals of $x$ are known, then $x$ lies in the decimal cylinder $[y, z]$ where $y=. d_{1} d_{2} \ldots d_{n}$ and $z=. d_{1} d_{2} \ldots d_{n}+10^{-n}$. In order to find the number of digits in the continued fraction expansion thus determined, let $y=\left[0 ; b_{1}, b_{2}, \cdots, b_{l}\right]$ and $z=\left[0 ; c_{1}, c_{2}, \cdots, c_{k}\right]$ be their continued fraction expansion. Then

$$
m(n, x)=\max \left\{i \leq \min (l, k): b_{j}=c_{j} \text { for all } j \leq i\right\}
$$

is the number of digits determined. In other words, $m(n, x)$ is the largest integer such that

$$
b_{n}(x) \subseteq c_{m(n, x)}(x)
$$

where $b_{n}(x)$ is the decimal cylinder of order $n$ containing $x$, denoted $[y, z]$ above, and $c_{m(n, x)}(x)$ is the continued fraction cylinder of order $m(n, x)$ containing $x$. Lochs [Lo] proved the following theorem:

Theorem 1.1. Let $\lambda$ denote Lebesgue measure on $[0,1)$. Then for $\lambda$-a.e. $x \in[0,1)$,

$$
\lim _{n \rightarrow \infty} \frac{m(n, x)}{n}=\frac{6 \ln 2 \ln 10}{\pi^{2}} .
$$

In 1999, Bosma, Dajani, and Kraaikamp [BDK] noticed that this problem could be rephrased in terms of dynamical systems. Define the maps $S x=10 x(\bmod 1)$ and $T x=\frac{1}{x}-\left\lfloor\frac{1}{x}\right\rfloor$ on $[0,1)$. Then $([0,1), \mathcal{B}, \lambda, S)$ and $([0,1), \mathcal{B}, \mu, T)$ are dynamical systems, where $\mathcal{B}$ is the Borel $\sigma$-algebra on $[0,1)$ and $\mu$ is the Gauss measure on $[0,1)$. Let the partitions $\mathcal{P}$ and $\mathcal{Q}$ be given by

$$
\mathcal{P}=\left(\left[0, \frac{1}{10}\right),\left[\frac{1}{10}, \frac{2}{10}\right), \cdots,\left[\frac{9}{10}, 1\right)\right), \mathcal{Q}=\left(\cdots,\left(\frac{1}{4}, \frac{1}{3}\right],\left(\frac{1}{3}, \frac{1}{2}\right],\left(\frac{1}{2}, 1\right)\right) .
$$

If we label $\mathcal{P}$ by $\left(p^{0}, p^{1}, \cdots, p^{9}\right)$ and $\mathcal{Q}$ by $\left(\cdots, q^{3}, q^{2}, q^{1}\right)$, then the decimal expansion of $x$ is achieved by iterating $x$ by $S$ and letting $d_{i}=k$ iff $S^{i-1} x \in$ $p^{k}$. Similarly the continued fraction expansion of $x$ is found by iterating $x$ by $T$ and setting $c_{i}=k$ iff $T^{i-1} x \in q^{k}$. Thus the expansions are actually the itineraries of $x$ for a certain partition in a certain dynamical system, 
and the intervals determined by the first $k$ terms of the expansion are the cylinder sets in the induced partitions $\bigvee_{i=0}^{k-1} S^{-i} \mathcal{P}, \bigvee_{i=0}^{k-1} T^{-i} \mathcal{Q}$

By using the Shannon-McMillan-Breiman Theorem [B] and the refinement of the partitions under application of their associated maps, they generalized Theorem 1.1 to a wider class of transformations, showing that

$$
\lim _{n \rightarrow \infty} \frac{m(n, x)}{n}=\frac{h(S)}{h(T)}
$$

where $h(S), h(T)$ indicate the entropy of the dynamical systems $([0,1), \mathcal{B}, \lambda, S)$, $([0,1), \mathcal{B}, \mu, T)$. Their proof assumes a certain regularity in the induced partitions, an assumption that was then dropped in the work of Dajani and Fieldsteel $[\mathrm{DF}]$, where it is proved that Lochs' Theorem is true for any two sequences of interval partitions on $[0,1)$ satisfying the conclusions of the Shannon-McMillan-Breiman Theorem. Their result can be immediately generalized to higher dimensional actions on $[0,1)$, using [Li] to yield a Shannon-McMillan-Breiman Theorem in this setting and then noting that the arguments in $[\mathrm{DF}]$ do not rely on the one-dimensionality of the action. Moreover, it is easy to see that the result of $[\mathrm{DF}]$ can be generalized to sequences of higher dimensional product partitions for which the projections of these partitions on each coordinate consist of intervals (see Final Remarks 2 ).

In this paper we consider the case of $[0,1)^{2}$. Let $\left([0,1)^{2}, \mathcal{B}, \lambda, T\right)$ and $\left([0,1)^{2}, \mathcal{B}, \mu, S\right)$ be two dynamical systems and $\mathcal{P}$ and $\mathcal{Q}$ two partitions of $[0,1)^{2}$. Given $\bar{x} \in[0,1)^{2}$, we can associate to it its $T, \mathcal{P}, n$ itinerary or equivalently, the element of $\bigvee_{i=0}^{n-1} T^{-i} \mathcal{P}$ in which it lies. We will call this element $p_{n}(\bar{x})$. We can do similarly for $S$ and $\mathcal{Q}$. We ask the same question as before: if we know the $T, \mathcal{P}, n$ itinerary of $\bar{x}$, what is the largest $m$ for which we know its $S, \mathcal{Q}, m$ itinerary, i.e. what is the largest $m$ such that $p_{n}(\bar{x}) \subseteq q_{m}(\bar{x}) ?$

In the following, we will phrase our result in terms of sequences of partitions. The situation where there are dynamical systems and partitions as mentioned above then yields the particular case where the sequences of partitions are given by $\bigvee_{i=0}^{k-1} S^{-i} \mathcal{P}$ and $\bigvee_{i=0}^{k-1} T^{-i} \mathcal{Q}$. In 1 dimension, all the partition elements were intervals. In 2 dimensions, the variety of partition shapes seen can be much greater and it turns out that the geometry of these shapes will play a role in the result. In the next section we will discuss our assumptions on the shapes and in section 3 we state and prove a 2-dimensional version of Lochs' Theorem.

\section{PARTitions}

We are interested in pairs of sequences of partitions, $\mathcal{P}=\left\{P_{n}\right\}, \mathcal{Q}=$ $\left\{Q_{n}\right\}$, of $[0,1)^{2}$. We will denote the elements of partition $P_{n}$ by $p_{n}^{i}$ and $Q_{n}$ by $q_{n}^{i}$. We denote by $\lambda$ the Lebesgue measure on $[0,1)^{2}$. There will be certain criteria that we will assume about these partitions. 


\section{Assumptions A}

$A 1$. For every $n, P_{n}$ consists of squares.

$A 2$. For every $n, Q_{n}$ consists of convex polygons of at most $k$ sides.

A3. There exists constants $R, S>0$ and $\beta \geq 2$ such that for every $n$ and every $i$,

$$
R \lambda\left(q_{n}^{i}\right) \leq\left(\text { diameter of } q_{n}^{i}\right)^{\beta} \leq S \lambda\left(q_{n}^{i}\right) .
$$

Assumption $A 3$ restricts the type of convex polygons that can be seen in $Q_{n}$. The isodiametric inequality [S] tells us that $\lambda\left(q_{n}^{i}\right) \leq\left(\text { diameter of } q_{n}^{i}\right)^{2}$, so the form seen in $A 3$ is natural. In order to get both sides of the inequality, it is not usually possible to use $\beta=2$. Under a very mild condition the following two lemmas tell us why we must have $\beta \geq 2$ and why, if $\beta$ exists, it is unique. As above, denote by $a_{n}(\bar{x})$ the element of a partition $A_{n}$ which contains $\bar{x}$.

Lemma 2.1. Let $\left\{A_{n}\right\}$ be a sequence of partitions of $[0,1)^{2}$ and suppose there exists an $\bar{x} \in[0,1)^{2}$ for which $\lim _{n \rightarrow \infty} \lambda\left(a_{n}(\bar{x})\right)=0$. Then there is no constant $S>0$ such that

$$
\text { (diameter of } \left.a_{n}^{i}\right)^{\alpha} \leq S \lambda\left(a_{n}^{i}\right)
$$

holds uniformly in $n$ and $i$ for any $\alpha<2$.

Proof. Suppose $\lim _{n \rightarrow \infty} \lambda\left(a_{n}(\bar{x})\right)=0$. It is always true that $\lambda\left(a_{n}(\bar{x})\right) \leq$ (diameter of $\left.a_{n}(\bar{x})\right)^{2}$. Now suppose that (diameter of $\left.a_{n}(\bar{x})\right)^{\alpha} \leq S \lambda\left(a_{n}(\bar{x})\right.$ ). We then have

$$
\lambda\left(a_{n}(\bar{x})\right) \leq\left(\left(\text { diameter of } a_{n}(\bar{x})\right)^{\alpha}\right)^{\frac{2}{\alpha}} \leq S^{\frac{2}{\alpha}} \lambda\left(a_{n}(\bar{x})\right)^{\frac{2}{\alpha}} .
$$

Hence

$$
S^{-\frac{2}{\alpha}} \leq \lambda\left(a_{n}(\bar{x})\right)^{\frac{2}{\alpha}-1} .
$$

For $\alpha<2$, the exponent on the right hand side is positive, so the right hand side tends towards zero as $n$ goes to infinity, which yields a contradiction.

Lemma 2.2. Let $\left\{A_{n}\right\}$ be a sequence of partitions of $[0,1)^{2}$ and suppose there exists an $\bar{x} \in[0,1)^{2}$ for which $\lim _{n \rightarrow \infty} \lambda\left(a_{n}(\bar{x})\right)=0$. Then there is at most one $\beta \geq 2$ for which there exists constants $R, S>0$ such that

$$
R \lambda\left(a_{n}^{i}\right) \leq\left(\text { diameter of } a_{n}^{i}\right)^{\beta} \leq S \lambda\left(a_{n}^{i}\right)
$$

holds uniformly in $n$ and $i$.

Proof. Suppose we have, for every $n$ and $i$,

$$
R \lambda\left(a_{n}^{i}\right) \leq\left(\text { diameter of } a_{n}^{i}\right)^{\beta} \leq S \lambda\left(a_{n}^{i}\right) .
$$

Suppose it is also the case that there exists constants $E$ and $\epsilon>0$ such that, for every $n$ and $i$,

$$
E \lambda\left(a_{n}^{i}\right) \leq\left(\text { diameter of } a_{n}^{i}\right)^{\beta+\epsilon} .
$$


Then from the right hand side of (1) we have in particular

$$
\text { (diameter of } \left.a_{n}(\bar{x})\right)^{\beta+\epsilon} \leq S^{\frac{\beta+\epsilon}{\beta}} \lambda\left(a_{n}(\bar{x})\right)^{\frac{\beta+\epsilon}{\beta}}
$$

and hence from (2)

$$
E \leq S^{\frac{\beta+\epsilon}{\beta}} \lambda\left(a_{n}(\bar{x})\right)^{\frac{\epsilon}{\beta}} .
$$

By assumption, $\lambda\left(a_{n}(\bar{x})\right)^{\frac{\epsilon}{\beta}}$ tends to zero as $n$ tends towards infinity, and we thus have a contradiction.

Similarly, suppose it is also the case that there exists a constant $F$ such that, for every $n$ and $i$,

$$
\text { (diameter of } \left.a_{n}^{i}\right)^{\beta-\epsilon} \leq F \lambda\left(a_{n}^{i}\right),
$$

where $\epsilon>0$ is such that $\beta-\epsilon>0$. Then from the left hand side of (1) we have in particular

$$
R^{\frac{\beta-\epsilon}{\beta}} \lambda\left(a_{n}(\bar{x})\right)^{\frac{\beta-\epsilon}{\beta}} \leq\left(\text { diameter of } a_{n}(\bar{x})\right)^{\beta-\epsilon}
$$

and hence from $(3)$

$$
R^{\frac{\beta-\epsilon}{\beta}} \leq F \lambda\left(a_{n}(\bar{x})\right)^{\frac{\epsilon}{\beta}} .
$$

As before, the right hand side goes to zero as $n$ tends to infinity, thus yielding a contradiction.

Recall that the partitions $Q_{n}$ consist of convex polygons $q_{n}^{i}$ of at most $k$ sides. We will be interested in the set of points in the polygons lying close to the boundary, defined as follows:

Definition 2.3. Let $q$ be a convex polygon. The frame of q of width $\delta$ is the set

$$
\mathcal{F}(q, \delta)=\{\bar{x}: \bar{x} \in q \text { and } d(\bar{x}, \partial q) \leq \delta\},
$$

where $\partial q$ is the boundary of $q$ and $d$ indicates the usual Euclidean distance on the plane.

The proportion of $q$ taken up by its frame is small when $\delta$ is small. The next lemma provides a bound that will be useful for us in the next section.

Lemma 2.4. Let $q$ be a convex polygon of at most $k$ sides such that

$$
(\text { diameter of } q)^{\beta} \leq S \lambda(q)
$$

for some constants $S$ and $\beta$. Then the proportion of $q$ taken up by its frame of width $\delta$ is bounded above by

$$
\frac{k S^{\frac{1}{\beta}} \delta}{\lambda(q)^{\frac{\beta-1}{\beta}}} .
$$


Proof. We are interested in $\frac{\lambda(\mathcal{F}(q, \delta))}{\lambda(q)}$. We can find an upper bound of the numerator by using $k$ (diameter of $q$ ) $\delta$, thus getting

$$
\frac{k S^{\frac{1}{\beta}} \lambda(q)^{\frac{1}{\beta}} \delta}{\lambda(q)}
$$

which can be rewritten to yield the above result.

Notice that if $q$ is a convex polygon, then the diameter is given by the largest length of the line segments connecting the vertices of $q$. This may be a side of $q$ or in the interior of $q$ (see figure below).
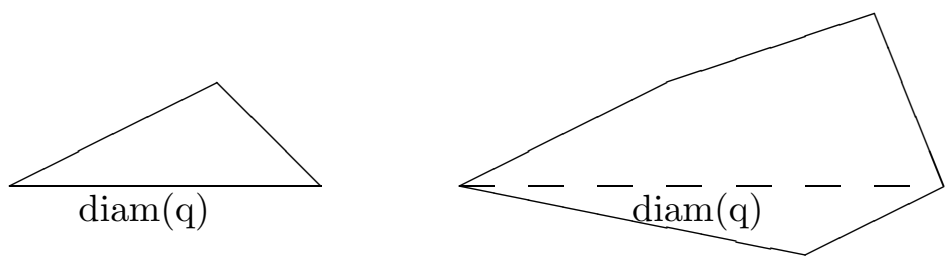

In either case, we want to define a quantity $h$ which will, in some sense, describe the "height" of $q$.

Definition 2.5. Let $q$ have vertices $\left\{v_{1}, \cdots, v_{k}\right\}$, where these are written in counterclockwise order.

If the diameter of $q$ is given by the length of the line segment $\mathbf{s}$ between $v_{j}$ and $v_{l}, l=j-1, j+1(\bmod k)$, i.e. by a side of $q$, then let

$$
h=\max \left\{d\left(v_{i}, \mathbf{s}\right)\right\} .
$$

Geometrically, this is the largest length of the line segments starting at the vertices $v_{i}$, drawn perpendicular to the diameter.

If the diameter of $q$ is given by the length of the line segment $\mathbf{s}$ between $v_{j}$ and $v_{l}, l \neq j-1, j+1(\bmod k)$, i.e. an interior line segment, then the diameter divides the polygon into two halves, which we denote the "top" and the "bottom". Let

$$
h_{1}=\max \left\{d\left(v_{i}, \mathbf{s}\right)\right\} \text {, where } v_{i} \text { is in the top half of } q
$$

and

$$
h_{2}=\max \left\{d\left(v_{i}, \mathbf{s}\right)\right\} \text {, where } v_{i} \text { is in the bottom half of } q .
$$

We then let

$$
h=h_{1}+h_{2} .
$$

By the above definition, the rectangle $r$ of base equal to the diameter of $q$ and height $h$ contains $q$ (see figure below). 

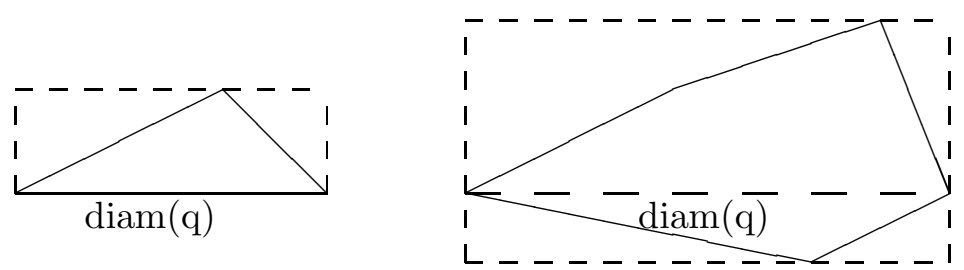

Lemma 2.6. Let $q$ be a convex polygon of diameter $d$ and let $r$ be the rectangle of base $d$ and height $h$. Then

$$
\lambda(r) \leq 2 \lambda(q) .
$$

Proof. In the case where $d$ is given by a side of $q$, inscribe a triangle in $q$ of base $d$ and height $h$. Then this triangle has area $\frac{1}{2} d \cdot h$, and $r$ has area $d \cdot h$. Thus $\lambda(r)=2 \lambda$ ( triangle). Yet clearly the area of the triangle is less or equal to the area of $q$, giving the result.

In the case where $d$ is not given by the side of $q$, inscribe two triangles in $q$ of base $d$ and heights $h_{1}$ and $h_{2}$. Their areas are $\frac{1}{2} d \cdot h_{1}$ and $\frac{1}{2} d \cdot h_{2}$, respectively. Thus $d \cdot h=d \cdot\left(h_{1}+h_{2}\right)=2$ ( area of the two triangles). Thus the area of the rectangle is twice of area of the triangles which is clearly less than or equal to the area of $q$.

The following obvious observation will be used in the next section.

Lemma 2.7. Let $p$ be a square of side length $x$ and $r$ be a rectangle of base $d$ and height $h$. If $x>h$ then $p$ is not contained in $r$.

We end this section with additional criteria for the partition sequences $\mathcal{P}=\left\{P_{n}\right\}$ and $\mathcal{Q}=\left\{Q_{n}\right\}$.

Definition 2.8. Let $\mathcal{P}=\left\{P_{n}\right\}$ be a sequence of partitions. Let $c \geq 0$. We say that $\mathcal{P}$ has entropy $c$ a.e. with respect to $\lambda$ if

$$
-\frac{\log \lambda\left(p_{n}(\bar{x})\right)}{n} \rightarrow c \text { for } \lambda \text {-a.e. } \bar{x} .
$$

\section{Assumptions B}

Let $\mathcal{P}=\left\{P_{n}\right\}$ and $\mathcal{Q}=\left\{Q_{n}\right\}$ be sequences of partitions of $[0,1)^{2}$ such that

$B 1$. For some constant $c>0, \mathcal{P}$ has entropy $c$ a.e. with respect to $\lambda$, and

$B 2$. For some constant $d>0, \mathcal{Q}$ has entropy $d$ a.e. with respect to $\lambda$.

If the sequence of partitions $\mathcal{Q}$ satisfies assumption $B 2$, it follows directly from Lemma 2.1 and Lemma 2.2 that there exists at most one $\beta \geq 2$ for which assumption $A 3$ holds. 
For ease of notation we will call $p_{n}(\bar{x})\left(\right.$ respectively $\left.q_{n}(\bar{x})\right)(n, \eta)$-good if

$$
2^{-n(c+\eta)} \leq \lambda\left(p_{n}(\bar{x})\right) \leq 2^{-n(c-\eta)}
$$

(respectively

$$
\left.2^{-n(d+\eta)} \leq \lambda\left(q_{n}(\bar{x})\right) \leq 2^{-n(d-\eta)}\right)
$$

\section{Main Theorem}

As before, $\lambda$ denotes the Lebesgue measure on $[0,1)^{2}$. Let $\mathcal{P}=\left\{P_{n}\right\}$ and $\mathcal{Q}=\left\{Q_{n}\right\}$ be sequences of partitions of $[0,1)^{2}$ satisfying assumptions (A) and (B) from section 2. For each $n \in \mathbb{N}$ and $\bar{x} \in[0,1)^{2}$, define

$$
m_{\mathcal{P}, \mathcal{Q}}(n, \bar{x})=\sup \left\{m: p_{n}(\bar{x}) \subseteq q_{m}(\bar{x})\right\} .
$$

Theorem 3.1. For $\lambda$-a.e. $\bar{x} \in[0,1)^{2}$,

$$
\frac{m_{\mathcal{P}, \mathcal{Q}}(n, \bar{x})}{n} \rightarrow \frac{\beta}{2(\beta-1)} \frac{c}{d},
$$

where $\beta \geq 2$ is the constant from assumption $A 3$.

The proof is in two parts. We will first show that

$$
\liminf _{n \rightarrow \infty} \frac{m_{\mathcal{P}, \mathcal{Q}}(n, \bar{x})}{n} \geq \frac{\beta}{2(\beta-1)} \frac{c}{d} \text { for } \lambda \text {-a.e. } \bar{x}
$$

and then

$$
\limsup _{n \rightarrow \infty} \frac{m_{\mathcal{P}, \mathcal{Q}}(n, \bar{x})}{n} \leq \frac{\beta}{2(\beta-1)} \frac{c}{d} \text { for } \lambda \text {-a.e. } \bar{x} .
$$

Together these give the result.

Proof. To prove (4), let $0<\epsilon<1$. For each $n$, let

$$
\tilde{m}(n)=\left\lfloor(1-\epsilon) \frac{\beta}{2(\beta-1)} \frac{c}{d} n\right\rfloor,
$$

where $\lfloor x\rfloor$ is the greatest integer less than or equal to $x$. Choose $\eta>0$ so small that $\zeta:=\epsilon \frac{c}{2}-\eta\left(\frac{c}{2 d}(1-\epsilon)+\frac{1}{2}\right)>0$. Consider the set of points

$$
D_{n}(\eta)=\left\{\begin{array}{ll} 
& p_{n}(\bar{x}) \text { is }(n, \eta) \text {-good, } \\
\bar{x}: \quad & q_{\tilde{m}(n)}(\bar{x}) \text { is }(\tilde{m}(n), \eta) \text {-good, and } \\
& p_{n}(\bar{x}) \nsubseteq q_{\tilde{m}(n)}(\bar{x})
\end{array}\right\} .
$$

If $\bar{x} \in D_{n}(\eta)$, then $\bar{x}$ lies in an element of $P_{n}$ which intersects at least 2 elements of $Q_{\tilde{m}(n)}$. Thus $\bar{x}$ must lie in the frame of $q_{\tilde{m}(n)}(\bar{x})$ of width $d$, where $d$ is the diameter of $p_{n}(\bar{x})$. Since $p_{n}(\bar{x})$ is a square, we know its diameter $d$ is $\sqrt{2}\left[\lambda\left(p_{n}(\bar{x})\right]^{\frac{1}{2}}\right.$. Since $p_{n}(\bar{x})$ is $(n, \eta)$-good, $\lambda\left(p_{n}(\bar{x})\right) \leq 2^{-n(c-\eta)}$, thus we know $\bar{x}$ must lie in the frame of $q_{\tilde{m}(n)}(\bar{x})$ of width $\sqrt{2} 2^{-\frac{n}{2}(c-\eta)}$.

We can thus bound the measure of $D_{n}(\eta)$ by the sum of all the frames of the $(\tilde{m}(n), \eta)$-good elements of $Q_{\tilde{m}(n)}$, of width $\sqrt{2} 2^{-\frac{n}{2}(c-\eta)}$. 
From Lemma 2.4, we know the proportion of an element $q_{\tilde{m}(n)}$ of the partition $Q_{\tilde{m}(n)}$ taken up by its frame of width $\sqrt{2} 2^{-\frac{n}{2}(c-\eta)}$ is bounded above by

$$
\frac{\sqrt{2} k S^{\frac{1}{\beta}} 2^{-\frac{n}{2}(c-\eta)}}{\lambda\left(q_{\tilde{m}(n)}\right)^{\frac{\beta-1}{\beta}}},
$$

where $k$ and $S$ are from assumptions (A).

Since $q_{\tilde{m}(n)}$ is $(\tilde{m}(n), \eta)$-good, we have that

$$
\lambda\left(q_{\tilde{m}(n)}\right)^{\frac{\beta-1}{\beta}} \geq 2^{-\tilde{m}(n) \frac{\beta-1}{\beta}(d+\eta)} .
$$

Hence

$$
\lambda\left(q_{\tilde{m}(n)}\right)^{\frac{\beta-1}{\beta}} \geq 2^{-(1-\epsilon) \frac{c}{d} \frac{n}{2}(d+\eta)} .
$$

Plugging this into the above, we see that the proportion of $q_{\tilde{m}(n)}$ taken up by its frame is bounded above by

$$
\begin{gathered}
\frac{\sqrt{2} k S^{\frac{1}{\beta}} 2^{-\frac{n}{2}(c-\eta)}}{2^{-(1-\epsilon) \frac{c}{d} \frac{n}{2}(d+\eta)}} \\
=\sqrt{2} k S^{\frac{1}{\beta}} 2^{\left[(1-\epsilon) \frac{c}{d} \frac{n}{2}(d+\eta)-\frac{n}{2}(c-\eta)\right]} \\
=\sqrt{2} k S^{\frac{1}{\beta}} 2^{-n\left[\epsilon \frac{c}{2}-\eta\left((1-\epsilon) \frac{c}{2 d}+\frac{1}{2}\right)\right]} \\
=\sqrt{2} k S^{\frac{1}{\beta}} 2^{-n \zeta}
\end{gathered}
$$

by the definition of $\zeta$ above. Thus the area of the frame of an $(\tilde{m}(n), \eta)$-good $q_{\tilde{m}(n)}$ is bounded above by

$$
\sqrt{2} k S^{\frac{1}{\beta}} 2^{-n \zeta} \lambda\left(q_{\tilde{m}(n)}\right)
$$

and thus

$$
\lambda\left(D_{n}(\eta)\right) \leq \sqrt{2} k S^{\frac{1}{\beta}} 2^{-n \zeta} .
$$

Then

which implies

$$
\sum_{n=1}^{\infty} \lambda\left(D_{n}(\eta)\right)<\infty
$$

$$
\lambda\left\{\bar{x}: \bar{x} \in D_{n}(\eta) \text { infinitely often }\right\}=0 .
$$

Since $\tilde{m}(n)$ goes to infinity as $n$ does, it follows that for $\lambda$-a.e. $\bar{x} \in[0,1)^{2}$, there exists an $N=N(\bar{x}) \in \mathbb{N}$ such that for all $n \geq N, p_{n}(\bar{x})$ is $(n, \eta)$ good and $q_{\tilde{m}(n)}(\bar{x})$ is $(\tilde{m}(n), \eta)$-good and $\bar{x} \notin D_{n}(\eta)$. But knowing that $p_{n}(\bar{x}) \subseteq q_{\tilde{m}(n)}(\bar{x})$ means that

$$
m_{\mathcal{P}, \mathcal{Q}}(n, \bar{x}) \geq \tilde{m}(n) .
$$

Thus for $\lambda$-a.e. $\bar{x} \in[0,1)^{2}$,

$$
\liminf _{n \rightarrow \infty} \frac{m_{\mathcal{P}, \mathcal{Q}}(n, \bar{x})}{n} \geq \liminf _{n \rightarrow \infty} \frac{\tilde{m}(n)}{n}=(1-\epsilon) \frac{\beta}{2(\beta-1)} \frac{c}{d} .
$$


Since $\epsilon$ was arbitrary, this gives the first part of our proof.

To prove part (5), let $\epsilon>0$. It is sufficient to show, for $\lambda$-a.e. $\bar{x}$, that

$$
\limsup _{n \rightarrow \infty} \frac{m_{\mathcal{P}, \mathcal{Q}}(n, \bar{x})}{n} \leq \frac{\beta}{2(\beta-1)} \frac{c}{d}(1+\epsilon) .
$$

Choose $0<\eta<d$ so small that $\zeta:=\epsilon \frac{c}{2}-\eta\left(\frac{c}{2 d}(1+\epsilon)+\frac{1}{2}\right)>0$. Take $\bar{x}$ from the set of full measure on which assumption (B) holds. Let

$$
\hat{m}(n)=\left\lceil\frac{\beta}{2(\beta-1)} n \frac{c}{d}(1+\epsilon)\right\rceil,
$$

where $\lceil x\rceil$ is the smallest integer larger than or equal to $x$.

Take $N=N(\bar{x})$ so large that

- for all $n \geq N, \lambda\left(p_{n}(\bar{x})\right) \geq 2^{-n(c+\eta)}$,

- for all $m \geq \hat{m}(N), \lambda\left(q_{m}(\bar{x})\right) \leq 2^{-m(d-\eta)}$, and

$-N>\frac{-\log \frac{1}{2} R^{\frac{1}{\beta}}}{\zeta}$.

We want to show that $p_{n}(\bar{x}) \nsubseteq q_{\hat{m}(n)+l}(\bar{x})$, for all $l \geq 0$ and $n \geq N$. As stated in Lemma 2.7, it is sufficient to show that the length of a side of the square $p_{n}(\bar{x})$ is larger than the value $h$ associated to $q_{\hat{m}(n)+l}(\bar{x})$, or equivalently, to the rectangle $r_{\hat{m}(n)+l}(\bar{x})$ that contains $q_{\hat{m}(n)+l}(\bar{x})$. Consider this value $h$. Suppose $l \geq 0$ and $n \geq N$. We know

$$
\begin{gathered}
h=\frac{\lambda\left(r_{\hat{m}(n)+l}(\bar{x})\right)}{\operatorname{diam}\left(r_{\hat{m}(n)+l}(\bar{x})\right)} \leq \frac{2 \lambda\left(q_{\hat{m}(n)+l}(\bar{x})\right)}{\operatorname{diam}\left(q_{\hat{m}(n)+l}(\bar{x})\right)} \\
\leq \frac{2}{R^{\frac{1}{\beta}}} \lambda\left(q_{\hat{m}(n)+l}(\bar{x})\right)^{\frac{\beta-1}{\beta}} \leq \frac{2}{R^{\frac{1}{\beta}}} 2^{-(\hat{m}(n)+l)(d-\eta) \frac{\beta-1}{\beta}} \\
\leq \frac{2}{R^{\frac{1}{\beta}}} 2^{-\hat{m}(n)(d-\eta) \frac{\beta-1}{\beta}} \leq \frac{2}{R^{\frac{1}{\beta}}} 2^{-\frac{n}{2} \frac{c}{d}(1+\epsilon)(d-\eta)} .
\end{gathered}
$$

On the other hand, the side length of $p_{n}(\bar{x})$ is $\lambda\left(p_{n}(\bar{x})\right)^{\frac{1}{2}}$ which is bounded below by $2^{-\frac{n}{2}(c+\eta)}$. We want to show that

$$
\text { side of } p_{n}(\bar{x}) \geq 2^{-\frac{n}{2}(c+\eta)}>\frac{2}{R^{\frac{1}{\beta}}} 2^{-\frac{n}{2} \frac{c}{d}(1+\epsilon)(d-\eta)}
$$

which is an upper bound for the value of $h$ for $q_{\hat{m}(n)+l}(\bar{x})$.

But $2^{-\frac{n}{2}(c+\eta)}>\frac{2}{R^{\frac{1}{\beta}}} 2^{-\frac{n}{2} \frac{c}{d}(1+\epsilon)(d-\eta)}$ can be rewritten as $\frac{1}{2} R^{\frac{1}{\beta}}>2^{-n \zeta}$, and we see that $N$ was chosen large enough so this would be true. Hence $m_{\mathcal{P}, \mathcal{Q}}(n, \bar{x})<\hat{m}(n)$ for $n \geq N(\bar{x})$ and part (5) follows.

We end this paper with some remarks concerning sequences of partitions $\mathcal{P}=\left\{P_{n}\right\}$ and $\mathcal{Q}=\left\{Q_{n}\right\}$ of $[0,1)^{d}$, where $d$ is an arbitrary positive integer. For ease of notation, we denote by $\lambda$ the Lebesgue measure on $[0,1)$ as well as the Lebesgue measure on $[0,1)^{d}$. Furthermore, we let $\bar{x}=\left(x_{1}, \cdots, x_{d}\right)$. 


\section{Final Remarks.}

1. Suppose the sequences of partitions $\mathcal{P}$ and $\mathcal{Q}$ satisfy the assumptions $B 1$ and $B 2$. It follows from the proof of Theorem 4 in [DF] that the inequality

$$
\limsup _{n \rightarrow \infty} \frac{m_{\mathcal{P}, \mathcal{Q}}(n, \bar{x})}{n} \leq \frac{c}{d} \text { for } \lambda \text {-a.e. } \bar{x}
$$

holds without imposing further conditions on the partition elements.

2. Suppose that for each $n, P_{n}$ and $Q_{n}$ are product partitions consisting of rectangles:

$$
P_{n}=P_{n}^{1} \times \cdots \times P_{n}^{d}, Q_{n}=Q_{n}^{1} \times \cdots \times Q_{n}^{d},
$$

where $P_{n}^{i}$ and $Q_{n}^{i}, 1 \leq i \leq d$, are interval partitions. Assume that for $1 \leq$ $i \leq d$, the sequences of partitions $\mathcal{P}^{i}=\left\{P_{n}^{i}\right\}$ and $\mathcal{Q}^{i}=\left\{Q_{n}^{i}\right\}$ have positive entropy $c_{i}$, respectively $d_{i}$, a.e. with respect to $\lambda$. Furthermore, assume that $Q_{n+1}$ refines $Q_{n}$ for each $n$. In general, the sequence of partitions $\mathcal{Q}$ does not satisfy assumption $A 3$. However, Theorem 4 in [DF] states that for $1 \leq i \leq d$,

$$
\frac{m_{\mathcal{P}^{i}, \mathcal{Q}^{i}}\left(n, x_{i}\right)}{n} \rightarrow \frac{c_{i}}{d_{i}} \text { for } \lambda \text {-a.e. } x_{i} .
$$

Since for each $n, Q_{n+1}$ refines $Q_{n}$, we have that

$$
m_{\mathcal{P}, \mathcal{Q}}(n, \bar{x})=\min _{1 \leq i \leq d} m_{\mathcal{P}^{i}, \mathcal{Q}^{i}}\left(n, x_{i}\right) .
$$

Hence, for $\lambda$-a.e. $\bar{x}$,

$$
\frac{m_{\mathcal{P}, \mathcal{Q}}(n, \bar{x})}{n} \rightarrow \min _{1 \leq i \leq d}\left\{\frac{c_{i}}{d_{i}}\right\}
$$

The condition in the previous remark stating that $Q_{n+1}$ refines $Q_{n}$ for each $n$ is not necessary as the following example shows.

Example. Let $\beta \geq 2$ and $c, d>0$ be given. Define the partition elements of $Q_{n}$ by

$$
q_{n}^{(i, j)}=\left[\frac{i}{\left\lfloor 2^{\frac{d(\beta-1) n}{\beta}}\right\rfloor}, \frac{i+1}{\left\lfloor 2^{\frac{d(\beta-1) n}{\beta}}\right\rfloor}\right) \times\left[\frac{j}{\left\lfloor 2^{\frac{d n}{\beta}}\right\rfloor}, \frac{j+1}{\left\lfloor 2^{\frac{d n}{\beta}}\right\rfloor}\right),
$$

$0 \leq i \leq\left\lfloor 2^{\frac{d(\beta-1) n}{\beta}}\right\rfloor-1,0 \leq j \leq\left\lfloor 2^{\frac{d n}{\beta}}\right\rfloor-1$, and define the partitions $Q_{n}^{1}$ and $Q_{n}^{2}$ by the relation $Q_{n}=Q_{n}^{1} \times Q_{n}^{2}$. One easily verifies that $\left\{Q_{n}^{1}\right\}$ and $\left\{Q_{n}^{2}\right\}$ have entropy $d_{1}=\frac{d(\beta-1)}{\beta}$ and $d_{2}=\frac{d}{\beta}$, respectively, a.e. with respect to $\lambda$. Define the partition $P_{n}=P_{n}^{1} \times P_{n}^{2}$ simply by partitioning $[0,1)^{2}$ into $\left\lfloor 2^{\frac{c n}{2}}\right\rfloor \times\left\lfloor 2^{\frac{c n}{2}}\right\rfloor$ squares of equal side length and notice that $c_{1}=c_{2}=\frac{c}{2}$. 
It is straightforward to show that the assumptions (A) and (B) are all satisfied. It follows from Theorem 3.1, that for $\lambda$-a.e. $\bar{x}$,

$$
\frac{m_{\mathcal{P}, \mathcal{Q}}(n, \bar{x})}{n} \rightarrow \frac{\beta}{2(\beta-1)} \frac{c}{d}=\min \left\{\frac{c_{1}}{d_{1}}, \frac{c_{2}}{d_{2}}\right\}
$$

However, if for instance $d_{1}$ or $d_{2}$ is small enough, $Q_{n+1}$ does not refine $Q_{n}$ for infinitely many $n$.

\section{REFERENCES}

[B] Billingsley, P. Ergodic Theory and Information, John Wiley and Sonc, Inc. ; New York (1965)

[BDK] Bosma, W., Dajani, K. , Kraaikamp, C. Entropy and counting correct digits, University of Nijmegen, report no. 9925, 1999, http: // www.math.kun.nl/onderzoek/reports/reports1999.html

[DF] Dajani, K. , Fieldsteel, A. Equipartition of Interval Partitions and an Application to Number Theory, Proc. AMS Vol 129, 12, (2001), 3453 - 3460.

[Li] Lindenstrauss, E. Pointwise theorems for amenable groups, Inventiones Mathematicae Vol 146 (2001), 259-295.

[Lo] Lochs, G. Vergleich der Genauigkeit von Dezimalbruch und Kettenbruch, Abh. Math. Sem. Hamburg, 27, (1964), 142-144.

[S] Stroock, D. A Concise Introduction to the Theory of Integration, Birkhäuser ; Boston (1994)

(K. Dajani) Department of Mathematics, Utrecht University, 3508TA Utrecht, The Netherlands

E-mail address: dajani@math.uu.nl

(M. de Vries) Faculty of Exact Sciences, Free University Amsterdam, 1081 HV, THE NETHERLANDS

E-mail address: mdvries@cs.vu.nl

(A.S.A. Johnson) Department of Mathematics and Statistics, Swarthmore College, Swarthmore, PA 19081

E-mail address: aimee@cc.swarthmore.edu 